\title{
Manejo adecuado de los residuos sólidos apoyados con las $\mathrm{TIC}^{1}$
}

\section{Appropriate management of solid waste Supported with TIC}

DOI: http://dx.doi.org/10.17981/cultedusoc.9.2.2018.09

Fecha de recepción: 05/06/2018. Fecha de aceptación: 30/08/2018

\author{
Cesar Fonseca ${ }^{2}$ \\ Agustín Lemus; Edilma Niño; Eligio Castilla; Juan Herrera; \\ Luis Castrillo; Nehemías Montes e Irina Castillo \\ IED Tomás Herrera Cantillo (Colombia) \\ entg7522@gmailcom
}

Para citar este artículo

Fonseca, C., Lemus, A., Niño, E:, Castilla, E., Herrera, J., Castrillo, L., Montes, N. y Castillo, I. (2018). Manejo adecuado de los residuos sólidos apoyados con las TIC. Cultura. Educación y Sociedad 9(2), 95-105. DOI: http://dx.doi.org/10.17981/cultedusoc.9.2.2018.09

\section{Resumen}

"El reciclaje es la transformación de las formas y los formatos habituales de los objetos, ya sea cartón, papel, lata, vidrio, algunos plásticos y residuos orgánicos, en materias primas" (Duran, 2003). El desarrollo de la presente investigación entonces es diseñar estrategias orientadas al Manejo adecuado de los residuos sólidos articulado a las diferentes áreas del conocimiento IEDTHC apoyado en TIC. La investigación se aborda desde una mirada cualitativa, de corte descriptivo y se emplea como técnica la entrevista, y las relatorías individuales. Los participantes fueron estudiantes de los grados $9^{\circ}$ de la institución educativa Tomás Herrera Cantillo. A modo de conclusión utilización del IEP como estrategias pedagógicas y el uso de las TIC, no nos permiten desarrollar metodologías con el fin de encontrar alternativas a la solución a las problemáticas ambientales que afectan a nuestra comunidad educativa.

Palabras clave: Reciclaje, residuos sólidos, aprendizaje significativo, estrategias, conocimientos científicos, TIC.

\begin{abstract}
The recycling is the transformation of the forms and the habitual formats of the objects Cardboard, paper, tin, glass, some plastics and organic residues, in raw materials. The development of this research is then to design strategies oriented to the proper management of solid waste articulated to the different areas of IEDTHC knowledge supported by ICT. The research is approached from a qualitative perspective, of a descriptive nature, and the interview is used as a technique, and the individual rapporteurs. The participants were students of the 9th grades of the Tomás Herrera Cantillo educational institution. As a conclusion of using the IEP as pedagogical strategies and the use of ICT, they do not allow us to develop methodologies in order to find alternatives to the solution to the environmental problems that affect our educational community.
\end{abstract}

Keywords: Recycling, solid waste, significant learning, strategies, scientific knowledge, TIC.

\footnotetext{
${ }^{1}$ Este artículo ha sido derivado del Programa de Fortalecimiento de la Cultura Ciudadana y Democrática CT+I a través de la IEP apoyada en TIC en el Departamento de Magdalena: CICLÓN. Desarrollado con docentes miembros del Grupo de investigación "Defensores Ecológicos" pertenecientes a la Institución Educativa Departamental Tomás Herrera Cantillo.

${ }^{2}$ Líder de investigación del grupo "Defensores Ecológicos".
}

- The author; licensee Universidad de la Costa - CUC. 


\section{Introducción}

De los problemas más significativos que presenta la humanidad se encuentra en duda alguna la degradación ambiental, del planeta, ésta problemática ha afectado sin duda alguna a las generaciones antiguas, a las presentes en una dimensión mayor, y de no tomar las medidas pertinentes a las futuras. Las causas de los problemas ambientales son muchos, y afecta cada escenario en donde se desenvuelven los individuos, social, político, de consumo, siendo este último quien denotan una fundamental influencia de la teoría económica, que resulta un factor decisivo, prioritario y fundamental, al momento de Contrarrestar problemas ambientales. (Herrera - Tapia, 2011; Hernández, 2011).

Los residuos sólidos se han convertido en un problema a nivel mundial en los últimos años; debido al exagerado aumento de una sociedad de consumo, sin conciencia ambiental, lo cual ha generado una contaminación ambiental y visual. En la actualidad la educación busca explora el desarrollo de seres humanos con valores y actitudes que les posibiliten una activa y constante participación en los contextos donde se desenvuelven. A partir de esta perspectiva la investigación es considerada como una práctica obligatoria encaminada al desarrollo de individuos analíticos y críticos que ayuden en el proceso de desarrollar nuevas y mejores sociedades (Dávila, 2012).

Metrópolis en todo el mundo cada día producen grandes cantidades de basura, algunos de éstos desechos son acumulados es espacios abiertos, donde abundan insectos y roedores, el resto termina por lo general en desechos sanitarios, los desperdicios que llegan ahí son desinteresantes y por lo general desagradables, como por ejemplo restos de comidas, trapos, desechos tóxicos, maderas, escombros, papeles y cartones que con el pasar del tiempo empiezan a descomponerse. Existen productos hechos a base de metal que se oxidan y que toman mucho más tiempo en descomponerse. Sin embargo, hay otros que lo hacen aún más tardío, como los productos hechos de vidrio y plástico. Mediante este proyecto se busca generar conciencia ciudadana en la disposición final de los residuos sólidos y en la reutilización de los mismos.

En el contexto en donde se encuentra la Institución Educativa Tomas Herrera Cantillo, en el corregimiento de peñoncito municipio de San Zenón, ubicado al norte con San Zenón, Oriente Pijiño del Carmen y Puerto Arturo, al oeste el brazo de Mompós, al sur con el horno Magdalena y Mompós Bolívar. Su territorio es plano, bajo, cenagoso, principalmente en las proximidades del río Magdalena. Existe una problemática ambiental latente en el Municipio el Lugar de disposición final de basuras se encuentra clausurado por lo que estas deben trasladarse para depositarlas en el botadero regional a campo abierto en el municipio de Santana, administrado por la Corporación Autónoma Regional del Magdalena, el cual tampoco ha sido habilitado aún, por tanto en este momento los residuos sólidos no cuentan con un manejo desde la administración municipal ni la cooperativa prestadora de servicios públicos.

La comunidad de San Zenón y sus corregimientos tiene una insipiente cultura de aseo y el destino final de los residuos sólidos termina siendo el rio o 
los solares cercanos al centro poblado, El crecimiento es ordenado del área urbana de los centros poblados y la falta de un sistema único de recolección de basuras, las vías urbanas y las rondas del rio y ciénagas funcionan como botaderos a cielo abierto; desaprovechando la oportunidad de hacer selección en la fuente y comercialización de los desechables reciclables.

Teniendo en cuenta lo anterior en la I.E.D. Tomas Herrera Cantillo enfatiza en el tratamiento adecuado de los residuos sólidos por tratarse de una institución de carácter ambiental; la comunidad educativa no ha tomado conciencia del daño ecológico que se causa al entorno, al no disponer racionalmente de estos. En la institución educativa diariamente se produce cantidades considerables de residuos sólidos como bolsas plásticas, latas, envases petts, cartón, papel, botellas de vidrios, bolígrafos inservibles, los cuales son depositados en canecas pertenecientes a la institución, pero, que al final por no existir en el municipio un lugar específico de disposición final de residuos, muchos de ellos son arrojados en la parte periférica de la institución educativa, por la falta de concientización de los estudiantes en el manejo adecuado de los mismos.

Estos residuos sólidos muchas veces son utilizados como elementos cortopunzante, por parte de ciertos alumnos quienes presentan una conducta violenta para amenazar a sus compañeros; además, ésta acumulación de residuos ha generado una contaminación ambiental dentro de la comunidad educativa, produciendo malos olores, presencia de roedores, reptiles, cucarachas, zancudos, entre otros, perturbando el buen proceso de enseñanza y aprendizaje por parte de los alumnos. Estas condiciones de insalubridad producen enfermedades como rinitis alérgica, dermatitis, parasitosis, dengue, enfermedades diarreicas, proliferación de mosquito, que se traducen en la falta de concentración de los estudiantes, deserción escolar y bajo rendimiento académico.

Actualmente, el crecimiento de las sociedades es cada vez es mayor, con todos los avances de la ciencia, la penetración delos mercados, las constantes transformaciones de supervivencia, es notorio activar las alarmas frente a todas las posibles problemáticas que esto pueda generar. Lo anteriormente planteado conlleva a estudiar uno de los factores que esto acarrea, como lo es la emergencia medioambiental, puesto que todos los recursos naturales que se encuentra disponibles, no soportan la demanda de acuerdo al porcentaje de crecimiento de la población. (Duran, 2003).

La creciente degradación ambiental que pone en riego toda situación vivencial en el planeta ha permitido que se busquen de manera acelerada alternativas de solución para preservar y proteger el entorno, contribuir a una cultura de protección del planeta y así mismo hacer uso consciente de los recursos sin poner en riesgo la permanencia de la vida. Según Duran:

El efecto invernadero está causando cambios ambientales y climáticos que han empezado a afectar la vida humana, además es evidente que las pautas actuales del suministro y consumo no son sostenibles. Muchas ciudades en el mundo sufren elevados niveles de contaminación, degradación de las comunidades y disfunciones sociales di- 
rectamente relacionadas con la falta de zonas verdes. P.P.13.

Unas de las estrategias que se han utilizado en los últimos tiempos para disminuir un poco la contaminación ambiental es el reciclaje, definido por Duran como "la transformación de las formas y presentaciones habituales de los objetos de cartón, papel, lata, vidrio, algunos plásticos y residuos orgánicos, en materias primas" (Duran, 2003). Es así como la utilización de las IEP como estrategias pedagógicas y el uso de las TIC, va a permitir desarrollar metodologías con el fin de encontrar alternativas de solución a las problemáticas ambientales que afectan a nuestra comunidad educativa.

El siguiente proyecto propende la utilización y clasificación de desechos sólidos como estrategias que posibiliten el aprendizaje, así los estudiantes tendrán la posibilidad de ver y manipu- lar elementos como el papel, el cartón, vidrio y residuos vegetales que posibiliten el desarrollo de conocimientos y al mismo tiempo crear sus propias definiciones y conceptos, partiendo del aprendizaje significativo, permitiendo que el individuo logre interpretar y comparar problemáticas ambientales que se dan en la sociedad en la que se desenvuelve, contrastando sus saberes previos con los conocimientos científicos (Palmero, 2004).

\section{Reciclaje}

El reciclaje puede ser definido como "la obtención de materias primas a partir de desechos, introduciéndolos de nuevo en el ciclo de vida y se produce ante la perspectiva del agotamiento de recursos naturales, macro económico y para eliminar de forma eficaz los desechos" (CONAM. s.f).

Tipo de residuos sólidos según la CEPIS (2001)

\footnotetext{
Residuos Sólido biodegradables: "Son aquellos residuos químicos o naturales que se descomponen fácilmente en el ambiente. En esta clasificación se encuentran las frutas, vegetales, restos de alimentos, madera y otros residuos que puedan ser transformados en materia orgánica". (CEPIS. 2001).
}

Residuos sólidos reciclables: "Son aquellos que no se descomponen fácilmente y pueden volver a ser utilizados en procesos productivos como materia prima. Entre estos residuos se encuentran: algunos papeles y plásticos, chatarra, vidrio, telas, partes y equipos obsoletos o en desuso, entre otros" (CEPIS. 2001).

Residuos sólidos inertes: "Son aquellos que no se descomponen ni se transforman en materia prima por ausencia de tecnología disponible en la región, o porque su degradación natural requiere grandes períodos de tiempo". (CEPIS. 2001).

Residuos sólidos comunes: "Que no son peligrosos y por su semejanza a los residuos domésticos son considerados como tales, se incluyen los provenientes de preparación de alimentos y todos aquellos que no pueden ser clasificado en las categorías A y B”. (CEPIS. 2001).

Residuos sólidos peligrosos: El término comprende a los desechos peligrosos derivados de todos los productos químicos tóxicos, materiales radiactivos, biológicos y de partículas infecciosas.

Fuente: Elaboración propia. (2018). 
El medio ambiente donde las personas nacen, crecen, se desarrollan, envejecen y mueren, es reconocido y revalorizado como muy importante en el mantenimiento de nuestra salud. "La ética ambiental se basa en el principio de solidaridad al concebir a la tierra como el espacio geográfico donde los seres humanos debieran compartir y disfrutar sus bienes, a través del aprovechamiento racional de los recursos naturales. (Secretaría de Medio Ambiente y recursos naturales s.f).

La educación ambiental es también la evaluación ética que se concreta sobre el ambiente como el bien y valor necesario para la humanidad contemporánea y para las futuras generaciones. Uno de los aspectos que identifican a la educación ambiental es su orientación hacia los valores, o sea, la inclusión ética de las relaciones entre el ser humano y su ambiente, y la consideración de éste como un bien a preservar. La finalidad de la educación ambiental es la aplicación de una ética fortalecida por un sistema de valores y comportamientos, entre los que se destacan cuestiones como la tolerancia, la solidaridad o la responsabilidad de la comunidad frente a los sistemas ambientales" (UNICEF, 2016).

Se hace necesario renovar la educación y orientarla al desarrollo sostenible, es por ellos que los países favorecerán la importancia y la intervención de estudiantes de las instituciones educativas en proyectos a niveles locales, regionales e internacionales, en donde estudien y aprendan como cuidar la naturaleza, el medio ambiente que los rodea, el agua y todos los recursos naturales, así mismo aprenderán sobre las consecuencias sociales y económicas que conlleva el manejo de éstos recursos. Teniendo en cuenta lo dicho surge el siguiente interrogante: ¿Cómo hablar de educación ambiental en la escuela? "La sensibilización creciente sobre el problema de la sustentabilidad influyó significativamente en la educación ambiental, haciéndola más compleja y demostrando sus vínculos con los problemas sociales, económicos, culturales y tecnológicos.

El problema es, entonces, lograr una masa crítica de población sensible a los problemas ambientales. Hoy no sólo es importante transmitir a los alumnos algunos conocimientos ambientales, sino educarlos para un desarrollo sustentable, promover el cambio de sus conductas y motivarlos a asumir responsabilidades personales y comunitarias referidas al medio ambiente" Programa Nacional Escuela y Comunidad, fundación Educambiente. Manual de Capacitación docente: Escuela, Ambiente y Comunidad (CONAM. s.f).

El programa de Tecnologías de la información y la comunicación (TIC) es el conjunto de tecnologías desarrolladas que incluye almacenamiento, recuperación, envío y recibimiento de información de un lugar a otro, o procesar la información para poder calcular resultados y elaborar informes, constituidas principalmente por la radio, la televisión y la telefonía convencional y por las Tecnologías de la información (TI) caracterizadas por la digitalización de las tecnologías de registros de contenidos (CEPIS. 2001). 
Usar las TIC constituye una diferenciación importante en la sociedad y en la educación, en las relaciones interpersonales y en la forma de difundir y generar conocimientos. Estas, van cambiando con el tiempo en la medida que se van dando los avances científicos, en un marco de globalización económica y cultural, contribuyendo a que los conocimientos estén a la vanguardia de los nuevos retos, provocando cambios de índole económicos social y cultural, dando oportunidades como: acceso al mercado de trabajo, la sanidad, la gestión económica y la comunicación entre otras. (UNICEF. 2016).

\section{Metodología}

La investigación es de tipo cualitativa, corte descriptivo. Al respecto (Tamayo, 2004) establece que la investigación de este tipo se distingue por la descripción, registro, análisis e interpretación de la naturaleza actual, composición o procesos de los fenómenos, se trabaja sobre las realidades de los hechos y sus características fundamentales. Tiene finalidad principal presentar un esclarecimiento considerable sobre una determinada situación.

\section{Escenarios y participantes}

Para darle solución a la problemática, la investigación tiene como participantes a los estudiantes de los grados $9^{\circ}$ de la institución educativa Tomás Herrera Cantillo, y las relatorías fueron aplicadas a 22 alumnos.

\section{Instrumentos de recolección de la} información

Los instrumentos utilizados para la recolección de la información para la presente investigación son:

Relatorías: Eficaz y útiles para la recolección de información en corto tiempo, como elemento de investigación, es una herramienta considerablemente aplicada de carácter cualitativa. La aplicación de este instrumento posee un grado objetivo. Mediante su desarrollo se pueden considerarse preguntas abiertas. También se empleó la observación, registrada en un diario de campo.

\section{Procedimiento}

La ejecución de la presente investigación está enmarcada en los siguientes momentos:

Momento (1): Elección del fenómeno a estudiar.

Momento (2): Hacer el sondeo diagnóstico de los objetos de interés.

Momento (3): Definición de las categorías de estudio: Residuos sólidos, TICs. Momento (4): Definición de los instrumentos de recolección de la información. Momento (5): Aplicación de la entrevista estructurada y los registros de observación.

Momento (6): Sistematización de la información a la luz de los datos recolectados y articulación con la teoría.

Momento (7): Generación de resultados. Momento (8): Socialización de los hallazgos encontrados.

\section{Resultados}

A continuación, se muestran los resultados derivados de las relatorías y la observación a los participantes. 


\begin{tabular}{|c|c|c|}
\hline $\begin{array}{c}\text { Categoría } \\
\text { de } \\
\text { estudio }\end{array}$ & $\begin{array}{c}\text { Pregunta } \\
\text { orientadora }\end{array}$ & Discurso de los actores \\
\hline
\end{tabular}

¿Considera que el manejo adecuado de los residuos sólidos que genera la institución contribuye de manera positiva a la comunidad educativa? ¿Por qué? "No porque en algunos casos los
No porque los alumnos tiran basura y no contribuyen con la comunidad", "No porque los residuos sólidos generan mucha contaminación", "No porque los residuos sólidos son malos para la salud", "Negativo porque además no hay un manejo adecuado para esos residuos", "No porque no nos beneficia a nosotros", residuos sólidos andan tirando por todo en la institución", "No porque la institución genera de manera positiva", "No porque muchos estudiantes no colaboran en recoger los residuos", "No porque la institución no genera de manera positiva a la comunidad "No porque la institución no contribuye de manera adecuada", "No, porque no lo hacen acá", "No porque los residuos sólidos generan mucha contaminación", "No porque no manejan los residuos como debe ser", No porque el manejo de residuos que se genera en esta situación no es positiva", "Si porque si se trabaja artística se transforma en arte", "Si porque si se maneja de manera adecuada podemos utilizarlo para hacer algo creativo", Si porque en muchas ocasiones se hacen jornadas de aseo para recolectar estos materiales", "Si porque contribuimos con la contaminación a la naturaleza dándole un buen uso al reciclaje", "Si porque si usamos de una manera muy bien cuidamos el medio ambiente", "En parte porque algunos proyectos que realizan en el colegio ayudan de una manera", "Si porque los residuos sólidos los utilizamos para hacer manualidades", "Si porque manejamos residuos como las botellas plásticas".

\section{Articulación y sistematización teórica}

Según lo que han respondido los estudiantes la mitad más tres, manifiestan que no están de acuerdo porque no se está realizando, es decir, actualmente no existe un programa de manejo adecuado para los residuos generados dentro de la institución, teniendo en cuenta que son grandes cantidades y estos manifiestan están tirados en todo el plantel educativo. Por ejemplo, según esta respuesta, "No porque no nos beneficia a nosotros", se percibe un grado de indiferencia y falta de cultura según dicho en la teórica del autor, Ramírez (s.f) "dice que los tres elementos claves son: Libertad, responsabilidad y la solidaridad teniendo como base el respeto por la naturaleza." Esto indica que debe haber solidaridad con nuestro propio planeta, sentido de pertenencia, no porque veo que alguien tira basura, deba adoptar la misma conducta teniendo como base fundamental el respeto, pero un respeto especial que es por la naturaleza, el medio donde te vales para sobrevivir, beneficiarte de él y mantenerte durante tu ciclo de vida. Es por esto que esta situación en la institución educativa de be ser ajena a ninguno de sus integrantes, y tomar medidas en el asunto, por eso surge la necesidad de crear un programa bajo una serie de actividades que fortalezcan principalmente la conciencia del publico frente a la problemática descrita, jornadas de sensibilización y concienciación, haciendo hincapié que es un problema de todos, y que desde lo personal cada uno aporta un grano de arena a la gran montaña de residuos sólidos que generan . De acuerdo a las respuestas afirmativas, tienen un fuerte y es que al poco buen manejo que dan a los residuos, los utilizan a favor de actividades didácticas desde el que hacer educativo.

Por otra parte el autor Reinfeld (2012) citado por (CANDELL, 2015) hace ver que un habitante en promedio genera un kilogramo de basura, haciendo que la producción de basura en nuestro país sea una cifra considerable, debido a que el número de habitantes aumenta con el tiempo y por lo tanto la producción de basura también. 


\begin{tabular}{|c|c|c|c|}
\hline $\begin{array}{l}\text { Residuos } \\
\text { Sólidos }\end{array}$ & $\begin{array}{l}\text { ¿De qué } \\
\text { manera } \\
\text { diaria uso y } \\
\text { tratamiento } \\
\text { a los residuos } \\
\text { generados por } \\
\text { la comunidad } \\
\text { educativa } \\
\text { fortalece tu } \\
\text { aprendizaje? } \\
\text { ¿Por qué? }\end{array}$ & $\begin{array}{l}\text { "Recolectando todo tipo de basura } \\
\text { como botellas plástico etc.", "No } \\
\text { porque no son beneficiosos porque } \\
\text { ya no sirven y son basura", "Si } \\
\text { porque así aprenderíamos más } \\
\text { que no debemos contaminar } \\
\text { el planeta", "Colaborando } \\
\text { con la limpieza y proteger la } \\
\text { integridad de la institución", } \\
\text { "De manera decorativa y } \\
\text { aprender a recolectar”, "No } \\
\text { porque no ayudaría a fortalecer } \\
\text { el aprendizaje”, "Yo le diera uso } \\
\text { reciclando botellas plásticas } \\
\text { haciendo figuras", "Yo le daría uso } \\
\text { al tratamiento de los recursos", } \\
\text { "Si porque con los residuos se } \\
\text { pueden trabajar artesanias", "No } \\
\text { porque en vez de recolectar lo que } \\
\text { hacen es tirarlos”, "No porque los } \\
\text { estudiantes botan la basura aun } \\
\text { sabiendo que no lo pueden hacer". }\end{array}$ & $\begin{array}{l}\text { Es el reciclaje, el que permite la } \\
\text { transformación de las formas y } \\
\text { presentaciones habituales de los objetos de } \\
\text { cartón, papel, lata, vidrio, algunos plásticos } \\
\text { y residuos orgánicos, en materias primas. } \\
\text { Es así como la utilización adecuada de los } \\
\text { diferentes desechos sirve como alternativa } \\
\text { de solución a las problemáticas ambientales } \\
\text { que afectan a nuestra comunidad } \\
\text { educativa. (Duran, 2003). } \\
\text { Lograr reciclar los residuos contribuye } \\
\text { a lo que expone Herrera (2014), generar } \\
\text { comportamientos responsables con el } \\
\text { entorno, en donde el hombre en su relación } \\
\text { plena con él puede destruir, pero a la vez } \\
\text { permitir hábitos sostenibles dependiendo de } \\
\text { la forma en la que le de uso a los recursos. }\end{array}$ \\
\hline TIC & $\begin{array}{l}\text { ¿Estás de } \\
\text { acuerdo en } \\
\text { crear una red } \\
\text { virtual que } \\
\text { te permita } \\
\text { interactuar } \\
\text { con la } \\
\text { comunidad } \\
\text { educativa en } \\
\text { pro de darle } \\
\text { manejo a } \\
\text { toda clase de } \\
\text { residuos en tu } \\
\text { contexto? ¿Por } \\
\text { qué? }\end{array}$ & $\begin{array}{l}\text { "Si para que más jóvenes se } \\
\text { unan y empiecen a recolectar", } \\
\text { "No porque cada uno debe ser } \\
\text { responsable con el daño que hace", } \\
\text { "Si positivo pues asi tendriamos } \\
\text { una institución limpia", "No } \\
\text { porque las personas no cuidan } \\
\text { lo que les dan", "Si porque hay } \\
\text { información que nos indica cómo } \\
\text { reducir residuos", "No porque } \\
\text { todos no tienen condiciones para } \\
\text { conectarse ya que no todos tienen } \\
\text { un computador o teléfono", "Si } \\
\text { porque sería bueno aprender } \\
\text { hacer cosas nuevas", "No porque } \\
\text { hay algunos que no manejan esta } \\
\text { red", "No porque no apoyan en } \\
\text { nada y hacen lo que ellos quieren", } \\
\text { "Si porque podemos interactuar } \\
\text { mucho mejor con todos así darle } \\
\text { un mejor manejo a los residuos } \\
\text { que nosotros mismo creamos", "No } \\
\text { estoy de acuerdo porque no todas } \\
\text { las personas son responsables", } \\
\text { "No porque aqui la gente no } \\
\text { tiene conciencia", "Aunque todo } \\
\text { sería mejor considero que mi } \\
\text { respuesta es sí porque todos } \\
\text { somos conscientes del daño que } \\
\text { causamos". }\end{array}$ & $\begin{array}{l}\text { Las TIC son herramientas teórico } \\
\text { conceptuales, soportes y canales que } \\
\text { procesan, almacenan, sintetizan, recuperan } \\
\text { y presentan información de la forma más } \\
\text { variada. Por lo tanto, poder articular } \\
\text { las nuevas tecnologías de la información } \\
\text { TIC para la búsqueda de soluciones a } \\
\text { cualquier problemática, como en este } \\
\text { caso el medio ambiental representa una } \\
\text { variación notable en la sociedad y a la } \\
\text { larga un cambio en la educación, en las } \\
\text { relaciones interpersonales y en la forma } \\
\text { de difundir y generar conocimientos. } \\
\text { Estas, van cambiando con el tiempo en } \\
\text { la medida que se van dando los avances } \\
\text { científicos, en un marco de globalización } \\
\text { económica y cultural, contribuyendo a que } \\
\text { los conocimientos estén a la vanguardia } \\
\text { de los nuevos retos, provocando cambios de } \\
\text { índole económicos social y cultural, dando } \\
\text { oportunidades como: acceso al mercado de } \\
\text { trabajo, la sanidad, la gestión económica } \\
\text { y la comunicación entre otras. (UNICEF. } \\
\text { 2016). }\end{array}$ \\
\hline
\end{tabular}




\begin{tabular}{|c|c|c|c|}
\hline TIC & $\begin{array}{l}\text { ¿Estás de } \\
\text { acuerdo } \\
\text { pertenecer } \\
\text { a esta red } \\
\text { virtual, la cual } \\
\text { te va permitir } \\
\text { promocionar } \\
\text { acciones que } \\
\text { contribuyan } \\
\text { a preservar } \\
\text { y mejorar } \\
\text { el medio } \\
\text { ambiente? }\end{array}$ & $\begin{array}{l}\text { "Si porque permite a animar a los } \\
\text { demás", "Así nos iría mejor", "Si } \\
\text { porque es para mejorar el medio } \\
\text { ambiente", "Si porque de esta } \\
\text { manera contribuiría a cuidar el } \\
\text { medio ambiente", "Si porque nos } \\
\text { brindan nuevos proyectos",", "Si } \\
\text { para tener mejor uso y preservar } \\
\text { el medio ambiente”, "Claro que sî", } \\
\text { "Si porque con grupos ayudamos } \\
\text { al medio ambiente a estar limpio", } \\
\text { "Si porque nos sirve para buscar } \\
\text { artes plásticos", "Si por que suena } \\
\text { interesante". }\end{array}$ & $\begin{array}{l}\text { La comunidad de San Zenón y sus } \\
\text { corregimientos tiene una insipiente cultura } \\
\text { de aseo y el destino final de los residuos } \\
\text { sólidos termina siendo el rio o los solares } \\
\text { cercanos al centro poblado, El crecimiento } \\
\text { es ordenado del área urbana de los } \\
\text { centros poblados y la falta de un sistema } \\
\text { único de recolección de basuras, las vías } \\
\text { urbanas y las rondas del rio y ciénagas } \\
\text { funcionan como botaderos a cielo abierto; } \\
\text { desaprovechando la oportunidad de hacer } \\
\text { selección en la fuente y comercialización } \\
\text { de los desechables reciclables." Teniendo } \\
\text { en cuenta lo anterior en la I.E.D. Tomas } \\
\text { Herrera Cantillo enfatiza en el tratamiento } \\
\text { adecuado de los residuos sólidos por } \\
\text { tratarse de una institución de carácter } \\
\text { ambiental y es desde el apoyo de las } \\
\text { tecnologías que la población se motiva de } \\
\text { manera diversa, y sobre todo aprendiendo } \\
\text { nuevas formas de cuidar el entorno. } \\
\text { (Diagnostico educativo, 2017) }\end{array}$ \\
\hline
\end{tabular}

Fuente: Elaboración propia. (2018).

\section{Discusión}

Los hallazgos encontrados con anterioridad se enmarcan en el estudio denominado: Programa: Diseño de estrategias orientadas al Manejo adecuado de los residuos sólidos articulado a las diferentes áreas del conocimiento IEDTHC apoyado en TIC en el Departamento del Magdalena. Y tiene en cuenta las siguientes consideraciones.

- Es de suma importancia realizar jornadas de concientización, pedagogización y sensibilización con el fin de que ellos entiendan la dimensión de la importancia en la disposición final de cada uno de los residuos que generan, no solo en la IED sino en sus hogares, contribuyendo así de manera general a todos los que hagan parte de esta.
- Mediar el buen manejo de residuos sólidos con el uso de los mismos en diferentes tipos de arte se convierte en una estrategia potencial y eficaz ya que según lo respondido, manifiestan que se sienten motivados, al saber que un desecho que es dañino se puede disponer finalmente para realizar artesanías y accesorios que ellos mismo fabrican, comercializan y utilizan.

- La clasificación final de los residuos sólidos es sumamente importante, así como también en el conocimiento del mismo, es por esto que comenzar con implementar en diferentes puntos estratégicos la ubicación de las canecas con la respectiva clasificación según la naturaleza del residuo. 
- Se debe socializar la plataforma educativa que se utilizara para la creación de la red virtual, con el fin de que todos pertenezcan a la misma, creen un perfil con el rol que quieran asumir, dándoles la potestad de tener un protagonismo propio dentro del programa, en esta podrán compartir las actividades que realizaran en pro del buen manejo de los residuos, la disposición final que les dé, videos tutoriales en donde compartan el proceso de fabricación a sus creaciones a base de material reciclable, compararan dentro de esta red así mismo, cuando tengan alguna clase de residuo solido reciclable con el fin de saber dentro de la comunidad quien está interesado en recibirla, ect.

- Se debe seguir motivando a la comunidad a través de talleres, mesa redonda, video conferencias, charlas en el buen manejo de los residuos sólidos. Está serie de actividades son oportunas ante un grandes cantidades de residuos que generan en sus hogares como en su comunidad educativa, en productos atractivos, funcionales y novedosos

\section{Referencias}

Alcaldía de Envigado. (2011). Guía para el adecuado manejo de los residuos sólidos y peligrosos. Documento recuperado de: http://www.ambientalex.info/ guias/Guia_manejo_residuos_sp.pdf Avendaño, I., Cortés, O. y Guerrero, H. (2015) Competencias sociales y tecnologías de la información y la comunicación como factores asociados al desempeño en estudiantes de básica primaria con experiencia de desplazamiento forzado Diversitas: Perspectivas en Psicología, 11(1) 13-36.
Candell, F. D. (2015). Creación de una planta de residuos sólidos en el cantón salinas, sector punta carnero. Santa Elena, Ecuador. Obtenido de http://repositorio.upse. edu.ec/bitstream/46000/1599/1/ DROUET\%20CANDELL\%20 FERNANDO.pdf

CEPIS. (2001). Marco conceptual de los residuos sólidos. Documento recuperado de: http://www.bvsde. paho.org/bvsars/e/fulltext/marco/ marco.pdf

CONAM. (s.f). Guía para la implementación del programa piloto de reaprovechamiento de residuos sólidos en huamanga, Pucallpa y Tingo María. Documento recuperado de: http://www.bvsde.paho.org/bvsacd/ cd27/guia-reapro.pdf

CORPAMAG. (2014). Plan de Gestión Ambiental Regional 2013-2027. Corporación autónoma Regional del Magdalena, Magdalena, Santa Marta. Obtenido de http://www. corpamag.gov.co/index.php/es/planeacion/plan-gestion-ambientalregional

Dávila, O. H. (2012). Plan de Desarollo Municipal. (A. Municipal, Ed.) San Zenón, Colombia. Obtenido de http:// cdim.esap.edu.co/BancoMedios/Documentos\%20PDF/sanzenonmagdalenapd20122015.pdf

Duran, D. (14 de Noviembre de 2003). Eco-Portal.Net. Obtenido de Educación ambiental: https://www. ecoportal.net/temas-especiales/ educacion-ambiental/escuela ambiente_y_comunidad.

Herrera-Tapias, B . (2011). El Estado Económico Constitucional de la Carta de 1991 frente al Derecho del Consumo. Revista Pensamiento Juridico 32(1), 247- 264. 
Hernandéz, D. (2011). Contabilidad ambiental: Fundamentos epistemológicos, humanistas y legales. Económicas CUC 32(1). 35-44.

Ley de Residuos Sólidos. (s.f). Cómo y porqué separar las basuras. Documento recuperado de: http://www.bvsde. paho.org/bvsacd/cd48/juntos.pdf

Palmero, M. L. (2004). LA TEORÍA DEL APRENDIZAJE SIGNIFICATIVO. (C. d. Hernández, Ed.) Pamplona, España. Obtenido de http://cmc.ihmc. us/papers/cmc2004-290.pdf
Tamayo, M. (2004). El roceso de la investigación científica. (N. Editores, Ed.) D.F México, México: LIMUSA S.A. Obtenido de http://www.intep.edu.co/ Es/Usuarios/Docentes/file/HelbertSalinas/Conocimiento_Cient.pdf

UNICEF. (2016). Cuadernillo de Medio Ambiente para comunidades indígenas. Argentila. Obtenido de https:// www.unicef.org/argentina/spanish/ manual-medioambiente-def2.pdf

UNICEF. (s.f). Experiencias urbanas de gestión integral de residuos en 10 municipios de Argentina. Documento recuperado de: https://www.unicef. org/argentina/spanish/EcoclubesbajaWEB.pdf 